ISSN 2693-2504

\title{
Congenital Chylothorax in Newborn with Trisomy 21
}

Journal of Bioscience \& Biomedical Engineering

Case Report

Ibrahim Kotbi ${ }^{1}$, Dr. Najia al hojaili ${ }^{* *}$, Dr. Attia alzahrani ${ }^{3}$, Dr. Helal Almalki ${ }^{4}$, Dr. Fakyria Madani ${ }^{5}$, Layla al abasi ${ }^{6}$, Dr. Nora Melaih ${ }^{7}$ and Dr. Abdullah Abdulrhman Alharbi ${ }^{8}$

${ }^{1}$ NICU Consultant, Maternity Children Hospital, Makkah

Saudi Arabia.

${ }^{2}$ NICU Consultant, Maternity Children Hospital, Makkah

Saudi Arabia.

${ }^{3}$ Head of department, NICU Consultant, Maternity Children Hospital, Makkah Saudi Arabia.

${ }^{4}$ Director of Maternity Children Hospital, Makkah Saudi Arabia, NICU Consultant.

${ }^{5}$ Head of pediatric department. Pediatric endocrine consultant, Maternity Children Hospital, Makkah Saudi Arabia.

${ }^{6}$ Seniour pediatric consultant, Maternity Children Hospital, Makkah Saudi Arabia.

${ }^{7}$ Saudi Board certified, Maternity Children Hospital, Makkah Saudi Arabia.

${ }^{8}$ Saudi Board, Maternity Children Hospital, Makkah Saudi Arabia.

*Correspondence authors

\author{
Dr. Najia alhojaili \\ NICU Consultant
}

Maternity Children Hospital

Makkah Saudi Arabia.

Submitted : 21 Jun 2021 ; Published : 3 Jul 2021

\begin{abstract}
Dawn syndrome Trisomy 21 means there's an extra copy of chromosome 21 in every cell. This is the most common form of Down syndrome, there is three types of Down syndrome.
\end{abstract}

Trisomy 21 which there is an extra copy of chromosome 21 in every cell. Mosaicism occurs when a child is born with an extra chromosome in some but not all of their cells. Translocation in this type of Down syndrome, children have only an extra part of chromosome 21. There are 46 total chromosomes. However, one of them has an extra piece of chromosome 21 attached.

Down syndrome is associated with a lots of complication like congenital heart disease. Vision. Hearing behavior and mental problem. Our case is Down syndrome with congenital chylothorax which is rare complication. Neonatal chylothorax results from the accumulation of chyle in the pleural space and may be either congenital or an acquired condition. Congenital chylothorax is most likely due to abnormal development or obstruction of the lymphatic system. It is often associated with hydrops fetalis. It can be idiopathic or may be associated with various chromosomal anomalies including Trisomy 21, Turner syndrome, Noonan syndrome, and other genetic abnormalities [1].

Treatment of chylothorax is multidisplenery need insertion of chest tube to decrease respiratory distress, diet management and pediatric surgery.

\section{Introduction}

Congenital chylothorax in neonates is serious problem .it was interfere with respiratory drive and need respiratory support, it can be diagnosed antenatal and mange. It associate with syndrome like Down syndrome, Noonan syndrome, turner. It can be acquired due to infection like pneumonia the chylous pleural effusion impair normal development of fetal lung by pressure effects and can lead to pulmonary hypoplasia Management of chylothorax is multidisplenary include neonatologist, pulmonologist, genetics, surgeon and dietitian. Post natal management include the support the ventilation, insert chest tube ,milk modification, drugs and the end surgical interfere about 1 in 1,000 babies born each year.

\section{Complication}

Complication of down syndrome its varies according to types, the most complication is congenial heart disease atioventricular septal defect, ventricular septal defect, Mitral valve prolapse, tetralogy of fallot. Vision problem like cataract, strabismus, keratoconus, cancer, Down syndrome increase incidence of germ cell tumor, either these blood or non-blood related, blood cancer increase in down syndrome up to $10-15 \%$ acute lymphoblastic leukemia is more common.

J B \& Bio Engine; $2021 \quad$ www.unisciencepub.com Volume 2 Issue 3 
Endocrine disease like hypothyroidism, diabetes are more common in Down syndrome so better to follow them in OPD with thyroid function test and random blood sugar twice per year. Gastrointestinal problem more common in Down syndrome like imperforated anus, duodenal atresia, Meckel diverticulum and celiac disease, constipation, so these complication will found in neonatal period or after childhood. Teething is problem in Down syndrome, gingivitis, tooth loose, oral hygiene and malocclusion also common.

Fertility is again problem in case of Down syndrome female reach to menopause earlier while male had problem with sperm development. Neurological delayed milestone in those babies with Down syndrome, there is several complication can occur in association with development of congenital chylothorax, these include pulmonary hypoplasia, malnutrition, hypernatremia and fluid imbalance. Complication due to prolong drain of chylothorax lead to impaired of lymphatic drainage and leads to infection.

\section{Our case}

Preterm 35 weeks delivered to 43 years old mother G5P4 with GDM. On metformin received one dose of dexamethasone. Baby delivered by cesarean section with APGAR score 6.7.7. in 1.5.15 Minutes. Initial steps done then intubated and connected to mechanical ventilator, baby had dimorphic feature in form of epicanthus fold, depressed nasal bridge, and short broad hands. He is hypotonic conscious.

Vital Sign was HR=130PPM, RR=40PPM, Temperatur36.5 c, saturation $=93 \%$

$1^{\text {st }}$ and $2^{\text {nd }}$ heart sound were normal, no murmur.

Abdomen is distended with ascites no organomegaly, genitalia were ambiguous

\section{Differential diagnosis}

1-Triasomy 21 with Transient tachypnea of newborn

1 -hydrops fetalis

2-pnemonia

3-liver impairment

4-Renal Impairment

\section{Investigation}

$\mathrm{CBC}=$ Complete blood count were normal.

Chemistry within normal Liver function test normal.

Albumine was corrected by albumin infusion.

Calcium was normal, total protein normal .LDH and Cholesterol were normal.

Pleural fluid Analysis was done in 27-4-2021, Amount $50 \mathrm{ml}$, Appearance was yellow turbid, $\mathrm{RBC}=3000$.WBC 6474(90\%monocyte, 5\% polymorph) Protein 31g/glucose $4.9 \mathrm{mmol} / \mathrm{L}$, Triglyceride $0.2 \mathrm{mml} / \mathrm{L}$ LDH $608 \mathrm{INU} / \mathrm{L}$ Culture no growth.

Repeated analysis was done on 30-4-2021 .Triglyceride 0.2 mmol/1 LDH 736 INU/L Amount 45 ML Appearance Amber turbid.

Blood culture was no growth.

Cerebrospinal fluid analysis RBCs (0), WBCs (100\%) monocyte, glucose normal, protein normal and culture no growth.
Urine Culture Staph maltphilia.

Chromosomal analysis sent and the baby was diagnosed with trisomy 21.

Echocardiogram showed PFO +Tiny PDA.

\section{Hospital Course}

Baby was admitted to NICU, started on IVF and antibiotics, chest X-ray showed Right side Plural effusion chest tube inserted and plural fluid sent to analysis and culture.

Baby developed neonatal seizure aborted by ant convulsion drug Phenobarbitone, Genetic Consultation was done they requested to do chromosomal analysis to rollout trisomy21.

Endocrine consultation was done unlikely to be congenial adrenal hyperplasia as 17-hydroxy progesterone was normal. Pulmonology consultation was done advice to send plural effusion to analysis and culture treat pneumonia to rollout hydrops fetalis exclude renal and liver impairment.

Baby was managed by continuous drain of pleural fluid, adequate ventilation .total parenteral nutrition dietary modification, albumin replacement. Octerotide was tried in this baby but still the effusion wasn't improve still the effusion. We used sildenafil in our patient when Octerotide had failed initial the effusion was decreased and we did chest $\mathrm{x}$-ray showed much improvement, but after while the effusion will accumulate again fax was sent to another pulmonologist advise no more intervention than what we did, the surgical interfere is the last think.

\section{Conclusion}

Congenital chylothorax is serious problem in neonate, early diagnosis and management improve the postnatal period. Management in postnatal include ventilator support, drain of chylous and sent to analysis and treated accordingly, dietary modification, drug therapy and the last one is surgery.

\section{Disclosure}

The authors report no conflicts of interest

\section{Reference}

1. Pub med Georgian Med News Nov; (236):74-77.

Copyright: (C2021 Dr. Najia alhojaili. This is an open-access article distributed under the terms of the Creative Commons Attribution License, which permits unrestricted use, distribution, and reproduction in anymedium, provided the original author and source are credited. 